\title{
Fear conditioning model predicts key temporal aspects of conditioned response production
}

\author{
JOHN P. MCGANN and THOMAS H. BROWN \\ Yale University, New Haven, Connecticut
}

\begin{abstract}
Five temporal aspects of the production of a conditioned response (CR) have been documented. Most of these phenomena were demonstrated using short-delay conditioning procedures, which are appropriate for eyeblink CRs. Relatively less is known about temporal aspects of CR production in longer delay conditioning procedures, which are common, for example, in studies of fear conditioning. Here, we show through computer simulations that our circuit-level model of fear conditioning predicts that these five time-domain phenomena should also be witnessed in longer delay conditioning procedures, provided that a suitable behavioral or neural probe is used. We explain how and why these phenomena emerge naturally from this general type of circuit-level model, which relies on intrinsic neuronal dynamics for time-domain computations, and we point out that the model furnishes a computational platform for Pavlov's original notion of a slowly spreading "wave of excitation." In accordance with Pavlov's thinking, these various temporal phenomena are captured using a contiguity-driven learning mechanism.
\end{abstract}

Pavlovian conditioning is a productive and powerful tool for studying the manner in which organisms learn temporal relationships among events (Kehoe \& Macrae, 1998; Moore \& Choi, 1997). In a typical delay conditioning paradigm, the conditioned stimulus (CS) is repeatedly paired with the unconditioned stimulus (US) such that there is a fixed latency or delay between the onset of the CS and the onset of the US. The timing of the conditioned response (CR) corresponds to the expected time of the US, furnishing evidence that this delay (i.e., the interstimulus interval [ISI]) is learned (Gibbon, Malapani, Dale, \& Gallistel, 1997; Gormezano, 1972; Kehoe \& Macrae, 1998; Mauk \& Ruiz, 1992). Learning of these latencies is regarded as "fundamental to classical or Pavlovian conditioning" (Gibbon et al., 1997).

Temporal learning in Pavlovian conditioning has been most thoroughly examined using the anticipatory eyeblink or the nictitating membrane responses as CRs. To obtain effective conditioning using these CRs, the ISIs are generally restricted to less than $1 \mathrm{sec}$ (Kimble \& Reynolds, 1967; Schneiderman, 1966; Schneiderman \& Gormezano, 1964). The effectiveness of different ISIs in supporting conditioning, sometimes called the ISI function, depends in part on the CR under investigation (Gormezano, 1972; Kehoe \& Macrae, 1998). Some studies that analyzed CRs other than the anticipatory eyeblink response have furnished evidence of temporal learning in the range of seconds to minutes (Davis, Schlesinger, \& Sorenson, 1989;

This work was supported by grants from the National Institutes of Health to T.H.B. and by a National Science Foundation Predoctoral Fellowship to J.P.M. The authors thank Susan Brandon, June-Seek Choi, James Moyer, and Allan Wagner for useful discussion. Correspondence should be addressed to T. H. Brown, Yale University, P.O. Box 208205, New Haven, CT 06520-8205 (e-mail: thomas.brown@yale.edu).
Ellison, 1964; Kehoe \& Macrae, 1998; Pavlov, 1927; Schneiderman, 1972). However, much of the longer delay conditioning data were produced on the basis of behavioral measures, such as conditioned freezing or salivation, that are not particularly well suited for investigating precisely the temporal aspects of conditioning. In particular, they lack the degree of temporal precision needed to constrain or inspire hypotheses about underlying neurobiological mechanisms. Most of our detailed knowledge about temporal aspects of CR production in Pavlovian conditioning therefore does not involve long ISIs.

The question therefore naturally arises as to whether or the extent to which the facts and rules regarding temporal aspects of CR production-as gleaned from short-delay conditioning procedures- - also apply to longer delay conditioning procedures. As noted earlier, the ISI function appears to depend at least in part on the particular CR, and the production of different CRs may engage different brain mechanisms (Kehoe \& Macrae, 1998). There is evidence, for example, that some CRs may require cerebellar circuitry but do not require an intact amygdala, whereas other CRs seem to require amygdalar circuitry but do not appear dependent on the cerebellum (Beggs et al., 1999; Kehoe \& Macrae, 1998; Maren, 1999; Thompson, 1988; Thompson \& Krupa, 1994; Weisz, Harden, \& Xiang, 1992; Weisz \& LoTurco, 1988). Conceivably, some of the basic facts and rules may not be the same for amygdala- versus cerebellum-dependent forms of conditioning.

In the present study, we examined predictions of our circuit-level model of fear conditioning (Tieu, Keidel, McGann, Faulkner, \& Brown, 1999), an amygdaladependent form of learning in which the conditioning procedure typically involves relatively long ISIs. Our neurobiologically inspired model was previously shown to learn accurately a wide range of ISIs. Computer simulations re- 
ported here show that an intrinsic feature of this general type of circuit model is that it predicts that five of the temporal aspects of CR production that were previously reported in conjunction with short-delay conditioning should also be evident in longer delay conditioning, provided that an appropriate response measure is used.

\section{Temporal Aspects of CR Production}

A number of facts or rules have emerged from shortdelay (short-ISI) classical conditioning procedures regarding the temporal aspects of CR production, five of which are summarized here. First, the CR onset precedes the expected US onset, and the latency of the CR onset decreases asymptotically across training (Frey \& Ross, 1968; Schneiderman, 1966; Schneiderman \& Gormezano, 1964; Suboski, 1967). Second, the peak amplitude of the CR seems timed to occur during the time of the US with an accuracy that may improve during training (Smith, 1968). The exact relationship between the CR peak and the US onset and offset is somewhat uncertain in these short-delay conditioning studies because the US duration is typically so short (commonly $50 \mathrm{msec}$ ). Third, training using mixed ISIs, where sequential conditioning trials may involve either of two different ISIs, can produce a double-peaked CR. In this case, the peaks of the two CRs correspond to the two ISIs used in training (Hoehler \& Leonard, 1976; Kehoe, Graham-Clarke, \& Schreurs, 1989; Millenson, Kehoe, \& Gormezano, 1977). Fourth, conditioning on one ISI and then shifting to a second ISI causes the CR latency to jump discretely from the latency of the first US to the latency of the second US (Boneau, 1958; Coleman \& Gormezano, 1971; Salafia, Martino, Cloutman, \& Romano, 1979). Several investigators have reported transition trials in which double-peaked CRs (corresponding to the two ISIs) are evident (Coleman \& Gormezano, 1971; Leonard \& Theios, 1967). Fifth, the averaged CR waveform becomes more spread out in time as the conditioning ISI increases (Smith, 1968, Figure 5; see also Gormezano, 1972, Figure 10).

While the most accurate data on temporal aspects of $\mathrm{CR}$ production come from short-delay conditioning procedures, there does exist some information about the timing of the CR in longer delay conditioning studies. For example, Pavlov's research on salivary conditioning in the dog showed that the $C R$ anticipated the expected time of the US, that the CR onset latency decreased over training trials, and that changing the ISI during conditioning resulted in a discrete jump in CR onset latency (Pavlov, 1927). Others have shown, using salivary conditioning, that the latency of the CR peak (time of maximal salivation rate) corresponded to the ISI used in training, which ranged from 5 to $16 \mathrm{sec}$ (Colavita, 1965; Ellison, 1964). Another study, using cardiac conditioning in dogs (Church \& Black, 1958), found that the latency of the maximum conditioned heart rate response was substantially longer when trained on a $20-\mathrm{sec}$ ISI ( $10.6 \mathrm{sec}$ mean latency) than when trained on a 5 -sec ISI ( $4.1 \mathrm{sec}$ mean latency). In both conditions, the onset of the CR preceded the expected time of the US.

Five studies suggest that information about the ISI is also acquired in fear conditioning. Two of these experiments involved conditioned enhancement of the unconditioned acoustic startle response (see J. S. Brown, Kalish, \& Farber, 1951). The first such study (Siegel, 1967) employed either a 4-sec or an 8-sec ISI during fear conditioning. When the animals were conditioned using a 4-sec ISI, testing was done at latencies from the CS onset of 2 , $3,4,5$, and $6 \mathrm{sec}$. The CS produced the maximum response when it preceded the acoustic startle stimulus by $4 \mathrm{sec}$. The startle responses were smaller when testing was done at a 3- or 5-sec latency, and the responses were even smaller when testing was done at a latency of 2 or $6 \mathrm{sec}$. This result has been interpreted (Davis et al., 1989) as indicating temporal learning. The interpretation is complicated, however, by the results of the conditioning done using the 8-sec ISI. In this case, testing done at latencies of $4,6,8,10$, and $12 \mathrm{sec}$ revealed little evidence of temporal learning.

Using this same basic paradigm, a second study (Davis et al., 1989) also furnished support for temporal learning in fear conditioning. Nine separate groups of rats received light-shock pairings at one of nine different conditioning ISIs (ranging from 0 to $51.2 \mathrm{sec}$ ). A 10th group, serving as the pseudoconditioning control, received unpaired presentations of the light and shock. During testing, each animal in all 10 groups was presented with the light followed by the acoustic startle stimulus. The startle stimulus was presented at nine different latencies from the CS onset, corresponding to the nine ISIs that were used for conditioning. These nine testing trial types were each presented 10 times, for a total of 90 nonreinforced CS presentations (extinction trials). Each group also received 20 startle-alone trials, which served as a baseline for evaluating the enhancing effect of the CS. For conditioning ISIs greater than $200 \mathrm{msec}$, the greatest CS-produced startle enhancement tended to occur when the acoustic startle stimulus was presented at a latency from the CS onset similar to the ISI that had been used for conditioning that particular animal.

A third study (Libby \& Church, 1975) that found evidence for temporal learning in fear conditioning employed the classical method of conditioned suppression of lever pressing (see Estes \& Skinner, 1941). One group of rats was trained with a $60-\mathrm{sec}$ CS (light) that always coterminated with the 0.5 -sec US (shock); a second group was trained with a 60 -sec CS during which shocks occurred at random with a mean frequency of once per $60 \mathrm{sec}$ (each 0.1-sec interval of the CS had an equal probability of beginning a US, so the animal might be shocked more than once or not at all during the CS). Conditioned suppression was measured for each 5-sec block of the CS, allowing the time course of the CR to be analyzed. The animals that were conditioned on a constant 60 -sec ISI showed a gradual increase in suppression over the course 
of the CS, peaking near the time of the US, suggesting that the subjects had learned the 60 -sec ISI. In contrast, the animals trained with randomly timed shocks during the CS showed a marked decline in suppression during the course of the CS, consistent with the declining probability of receiving a shock in the part of the CS that remained. Additional experimental groups showed that when the duration of the CS was randomized (thus, the length of time since its onset did not help predict the time of the next shock), the subjects' level of fear was constant after the transient CS onset effect shown by all groups.

The preceding three studies entailed cued fear conditioning, where a discrete CS furnished temporal information about the US onset. Two additional experiments used one-trial contextual fear conditioning, examining temporal encoding by varying the interval between placement of the rat in the context and the time of the shock US (Bevins \& Ayres, 1995; Maes \& Vossen, 1992). Analysis of the time course of freezing during subsequent exposure to the conditioned context in these studies revealed evidence that the subjects froze more during the time period that had previously included the US than during other time periods, thus suggesting temporal learning in contextual fear conditioning.

\section{Conceptual and Computational Model}

Mounting evidence suggests that fear conditioning depends on the lateral nucleus of the amygdala (ALa) (for reviews, see Fanselow \& LeDoux, 1999, LeDoux, 1995, Maren, 1999, and Maren \& Fanselow, 1996). A popular hypothesis is that conjoint and converging activity in CSand US-driven synaptic inputs to ALa neurons can result in associative long-term potentiation (LTP), a Hebbian form of synaptic plasticity (Barrionuevo \& T. H. Brown, 1983; Chapman, Kairiss, Keenan, \& T. H. Brown, 1990; Kelso \& T. H. Brown, 1986; Kelso, Ganong, \& T. H. Brown, 1986; Levy \& Stewart, 1979; Magee \& Johnston, 1997; Maren, 1996, 1999). The idea is that, after the induction of associative LTP, the synapses associated with the CS-driven cells would be sufficiently strengthened to fire the postsynaptic neurons, giving rise to conditioned fear responses. Single-unit studies in ALa done in freely moving rats are consistent with this interpretation (Quirk, Repa, \& LeDoux, 1995). The output of ALa is believed to bring about certain fear responses, in part via its influence on the central nucleus of the amygdala (ACe) (Applegate, Kapp, Underwood, \& McNall, 1983; Canli \& T. H. Brown, 1996; Kapp, Frysinger, Gallagher, \& Haselton, 1979; Kapp, Whalen, Supple, \& Pascoe, 1992).

We previously developed a neurobiologically inspired conceptual model of the role of perirhinal cortex (PR) and ALa in generating appropriately timed conditioned fear responses at relatively long ISIs (Faulkner, Tieu, \& T. H. Brown, 1997). The model, schematized in Figure 1, makes use of the dynamics of several of the main cell types found in these brain regions (fast-spiking, regular-spiking, and late-spiking cells) and their interconnections (Faulkner \& T. H. Brown, 1999). Central to the model is the existence of late-spiking cells, a common cell type in PR that can produce a spike train delayed by $1 \mathrm{sec}$ or more from the onset of a train of synaptic inputs or a depolarizing current step (Beggs, Moyer, McGann, \& Brown, 2000; Faulkner \& T. H. Brown, 1999). Chains of various lengths composed of these and other types of neurons can potentially delay the spread of neural activity through the circuit by large amounts of time.

In our conceptual model, CS information flows from visual, auditory, and other sensory cortices to PR, then from $P R$ to ALa along chains of regular- and late-spiking neurons that accumulate various delays from the CS onset (Figure 1). Because of recurrent inhibition and the strong spike frequency adaptation of neurons in ALa, CS-driven activity at the end of each chain (in ALa) is limited to a brief window of firing, or activity window, which begins at some delay from the CS onset (depending on the chain through PR) and terminates when the corresponding cells in ALa cease firing. Information about the US travels with minimal delay through other pathways and also reaches ALa (Romanski, Clugnet, Bordi, \& LeDoux, 1993; Shi \& Davis, 1999). When there is co-occurrence of CS- and US-driven input to an ALa neuron, a Hebbian mechanism (T. H. Brown \& Faulkner, 1997; T. H. Brown, Kairiss, \& Keenan, 1990; Kelso et al., 1986) is presumed to potentiate the CS-driven synapses onto that neuron (Maren, 1996, 1999; Maren \& Fanselow, 1996). After sufficient potentiation, CS-driven activity is able to induce appropriately timed firing in ALa. The lateral amygdala in turn mediates the behavioral response in part through its projections to ACe (Krettek \& Price, 1978; Stefanacci et al., 1992).

We created a scaled-down computational implementation of this conceptual model that uses realistic model neurons whose dynamical firing properties are matched to our physiological data and that are connected in a manner suggested by our preliminary anatomical studies (Faulkner \& T. H. Brown, 1999). The strengths of the plastic synapses are modified according to a BCM-type plasticity rule (Bienenstock, Cooper, \& Monro, 1982), so that a small amount of postsynaptic activity causes long-term depression (LTD) in all synapses with simultaneous presynaptic activity, whereas a large amount of postsynaptic activity causes LTP in these synapses (Malenka, 1995).

This initial version of the computational model does not yet include recurrent circuitry or inhibitory neurons, so information flow is purely feedforward and the temporal activity windows terminate as a result of the accommodation of individual model neurons. In a previous detailed report of the model (Tieu et al., 1999), we demonstrated that it showed pairing-specific learning and was capable of encoding temporal intervals from tenths of seconds to tens of seconds. The upper ISI limit that we have explored is $20 \mathrm{sec}$, but larger networks can encode longer intervals (at the expense of longer computer simulation times).

The current generation of the computational model allows for only a single CS, which is represented simply by a constant input (corresponding in time to the CS duration) 


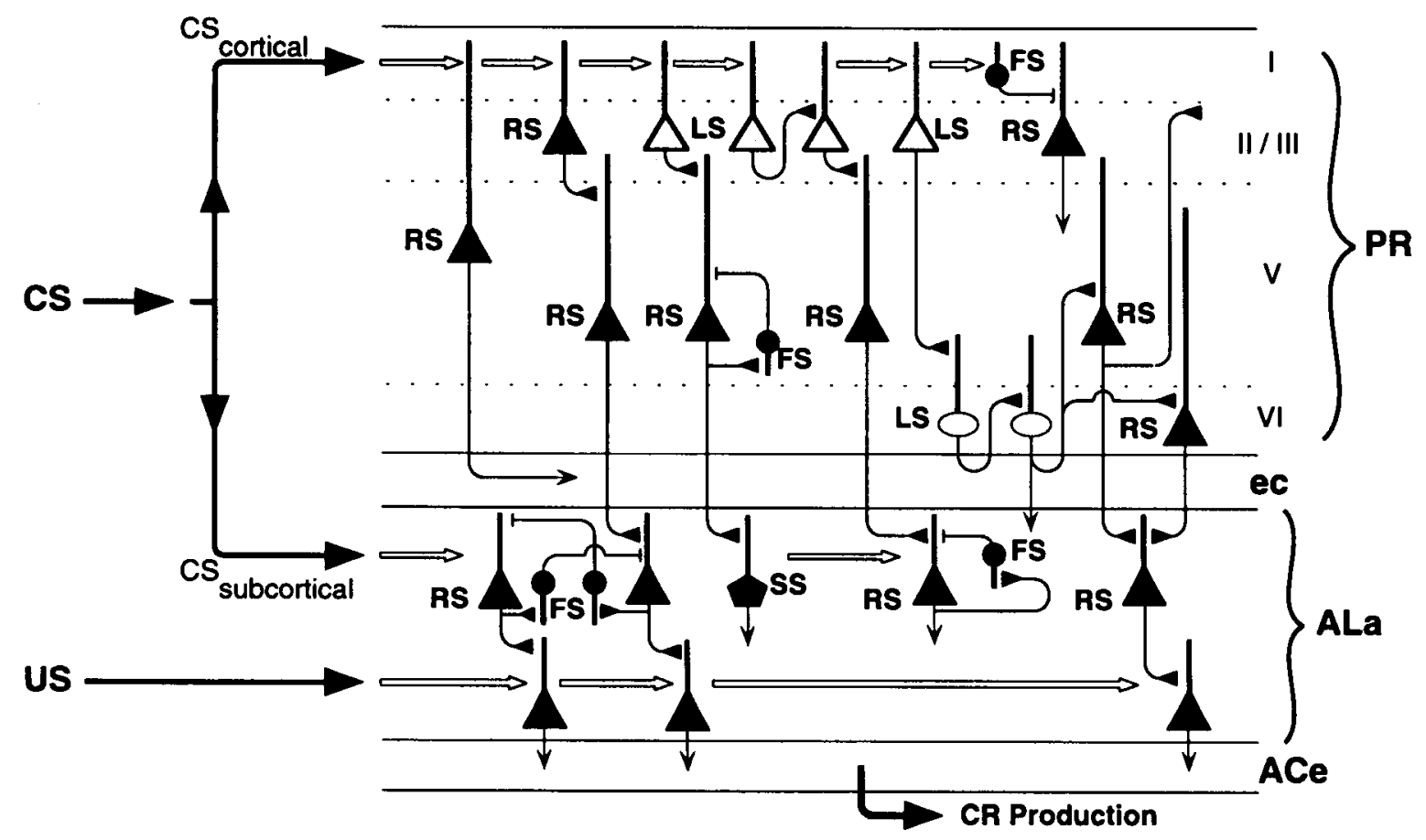

Figure 1. Circuit-level conceptual model of information processing and fow during aversive conditioning. Excitatory synapses are indicated by small triangular endings and inhibitory synapses by a flat ending. CS information enters via both a cortical and a subcortical path, but only the cortical input $\left(\mathrm{CS}_{\text {cortical }}\right)$ is presumed to encode time (CS-US interval information). CS and US information flow are indicated by filled arrows (before entering the PR-AM system) and by hollow arrows (after entering the system). CS input to layer I activates various time-delay chains in PR. CS-produced synaptic activity in ALa that coincides with US-produced activity in the same ALa neuron(s) results in associative LTP of the CS-associated input, thereby enhancing the strength of the response to the $\mathrm{CS}$ at the appropriately timed latency. Inhibition serves several functions, including vector "normalization," creation of activity windows, and competitive learning in ALa. The output of ACe is presumed to mediate certain indices of fear or arousal to generate the observed CR. Abbreviations are as follows: CS, conditioned stimulus; US, unconditioned stimulus; $C R$, conditioned response; PR, perirhinal cortex; ec, external capsule; $A L a$, lateral nucleus of the amygdala; ACe, central nucleus of the amygdala; LS, late firing cell; RS, regular firing cell; FS, fast firing cell; SS, single spiking cell. Modified from Tieu et al. (1999).

to the first cell in each PR chain. The US is represented by a constant input (corresponding in time to the US duration) to ALa neurons as illustrated in Figure 1. Its strength is sufficient to drive the model ALa neurons into the high end of their dynamic ranges and above the threshold for synaptic potentiation (Tieu et al., 1999). In these simulations, the dependent variable is the neural output from ALa (see Simulation Methods section). Thus, the measured variable is a "neural CR" rather than a simulated behavior.

To mimic behavioral conditioning paradigms, the computational model includes two standard trial types: acquisition trials, in which the CS and US are both presented, and extinction or "probe" trials, in which the CS is presented alone. For convenience, the model also makes use of "observation" trials, in which the CS can be presented alone without allowing alterations in the plastic synapses. These trials allow the experimenter to observe the CSdriven response without introducing testing-produced changes.

\section{Simulation Methods}

Three different simulations were designed to evaluate the temporal form of the model's CR in the behavioral paradigms described above. For these simulations, we used the same computer program and network architecture as reported elsewhere (Tieu et al., 1999), with minor parametric variations that affected the relative rates and exact conditions under which synapses undergo LTP and LTD.

In the conceptual model, the output of ALa controls the input to $\mathrm{ACe}$ and the output of $\mathrm{ACe}$ is linked to the expression of conditioned fear (see introduction). To economize on simulation times, in the present study, we excluded ACe and analyzed only the output from ALa. Of course, the behavioral fear response could be delayed and might exceed the duration of the "neural CR" in ALa. We are assuming that a suitable behavioral index, such as conditioned reflex facilitation (J. S. Brown et al., 1951; Lam, Wong, Canli, \& T. H. Brown, 1996; Weisz et al., 1992; Weisz \& LoTurco, 1988; Weisz \& McInerney, 1990; Weisz \& Walts, 1990), will reveal a correspondence to the neural CR. The model never exhibits CS-produced output prior to training or following pseudoconditioning (Tieu et al., 1999). Therefore, any output from ALa is the result of learning. We operationally defined the latency of the neural $C R$ onset in each trial to be the latency of the first spike to occur in the output from ALa in that trial. The latency of the $C R$ peak was operationally defined to be the average latency of the CS-generated output spikes in ALa. The average seemed to be an appropriate measure, because the frequency distribution of spike latencies appeared Gaussian and the mean was more reliable than the mode.

The random synaptic noise in the model (Tieu et al., 1999) can produce substantial variability in both learning and performance. 

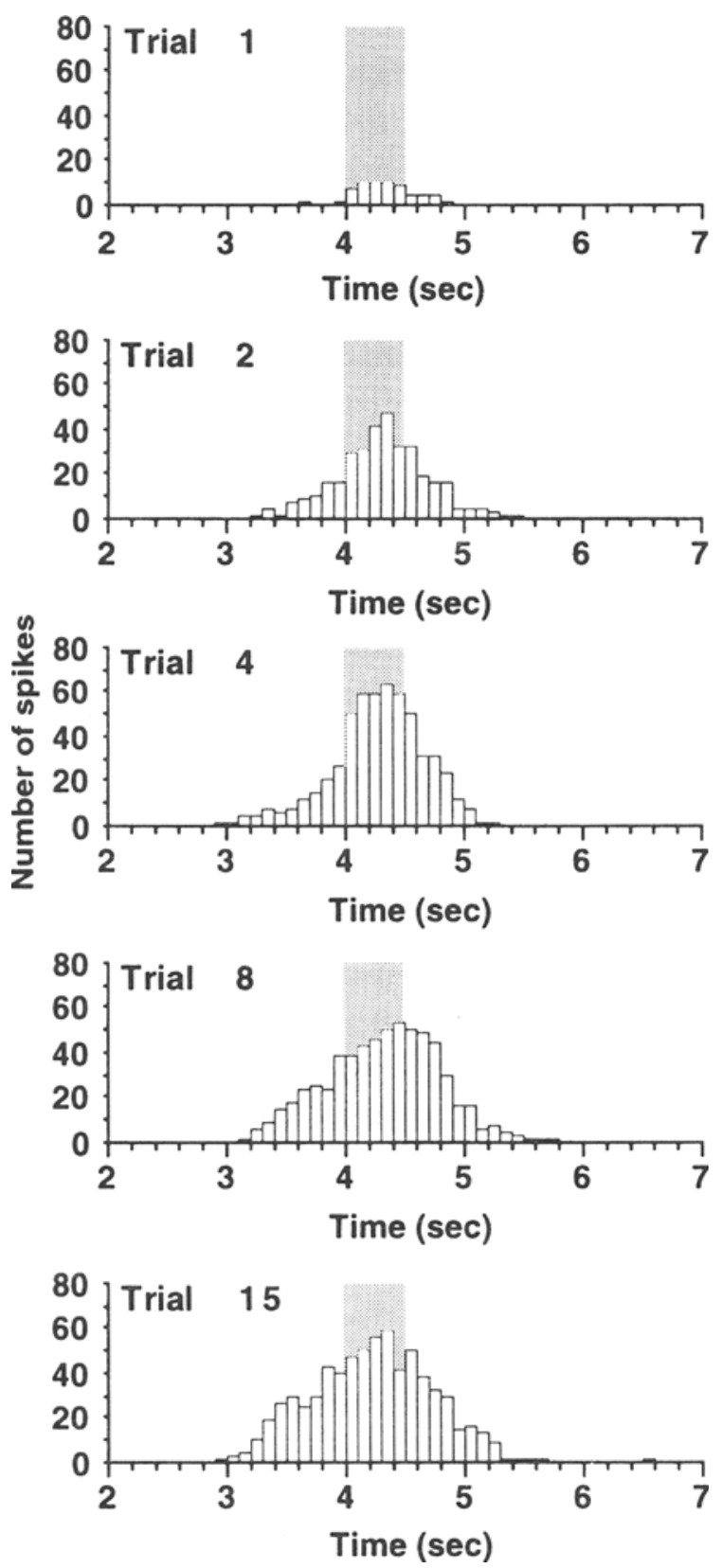

Figure 2. Development of the conditioned neural response (CR) across acquisition trials. Each panel shows a histogram of the latencies of the spikes that constitute the output from lateral amygdala during an observation trial presentation of the CS alone for 8 sec (this trial type permits the measurement of the conditioned response without allowing learning-related changes to occur; see Simulation Method section). In descending order, the panels show the $C R$ after $1,2,4,8$, and 15 acquisition trials. The histograms represent the pooled results of six statistically independent simulations. During training, the US was presented for $0.5 \mathrm{sec}$, beginning $4 \mathrm{sec}$ after the CS onset. Note that the peak of the distribution corresponded to the expected time of the US in each case (indicated by the shaded box) and that the onset of the response typically preceded the expected onset of the US. In addition, the average latency of CR onset decreased across acquisition trials, which is visible in these histograms of pooled data as the increase in the number of spikes that precede the expected US onset across trials. See Figure 3 for a quantitative analysis of the CR topography changes across trials.
Because of this randomness, repeated simulations (using a different random seed each time) gave somewhat different results. To obtain a more representative sample of the model's behavior, we therefore ran each type of simulation six times and combined the results. These different simulations are analogous to different experimental subjects. For the numerical values of the neural CR peak and onset latencies, such as those plotted in Figure 3, we averaged the measurements on corresponding trials across runs (for most data points, standard errors were too small to represent in Figure 3). For continuous representations of the temporal form of the CR, such as the histograms shown in Figures 2, 4, and 5, we pooled the data from corresponding trials across runs.

The first simulation type involved simple acquisition, in which the CS was paired with the US on 15 consecutive trials, enough pairings to approach asymptotic performance. The purpose of these simulations was partly to determine the necessary number of conditioning trials in the subsequent studies. The CS onset to US onset ISI was $4 \mathrm{sec}$, the CS duration was $8 \mathrm{sec}$, and the US duration was $0.5 \mathrm{sec}$. Recall that the neural CR was defined as CS-produced spikes output from ALa. These acquisition data were collected using observation trials (in which the CS was presented alone without allowing synaptic modifications to occur) after each conditioning trial. The testing trials performed during acquisition, therefore, could not cause extinction (it was possible to observe without changing the system). The CS duration on these observation trials was $8 \mathrm{sec}$.

In a second type of simulation, we examined CS-produced neural output from ALa after conditioning using two different ISIs on alternating trials. In this mixed ISI conditioning, the model was trained for a total of 20 trials, in which the ISI alternated between 5 and $15 \mathrm{sec}$. The CS duration was $18 \mathrm{sec}$, and the US duration was $0.5 \mathrm{sec}$. Testing again used observation trials after each conditioning trial, but, in this case, the CS duration was $18 \mathrm{sec}$. To control for a possible order effect of the trial type, the six runs of this simulation were counterbalanced so that the 5-sec ISI was presented first on three of the runs and the 15-sec ISI was presented first on the other three.

A third type of simulation examined the neural CR when the ISI was shifted during conditioning. The circuit was first trained for 15 trials on a 4-sec ISI, followed by 15 additional training trials on a 12-sec ISI. In this simulation, the CS lasted $15 \mathrm{sec}$, the US lasted $0.5 \mathrm{sec}$, and the CR was recorded after every training trial using an observation trial, again using a $15-\mathrm{sec}$ CS. To evaluate the possible effects of the direction of shift, in a separate set of simulations, the circuit was first trained with a 12-sec ISI and then trained with a 4 sec ISI. In this second set of simulations, nothing was changed except the order of conditioning.

\section{Results}

The simulations of our circuit-level model suggested that the five key temporal hallmarks of CR timing that have been observed during short-delay conditioning should also occur during longer delay conditioning.

CR onset precedes the expected time of the US. Figure 2 shows the development of the neural CR across trials using a 4-sec ISI. Recall that the data presented in Figure 2 are the pooled results of six independent simulations. Visual inspection of Figure 2 reveals that the neural CRs were anticipatory. The mean onset latency $( \pm S E)$ was $3.36 \pm 0.05 \mathrm{sec}$ across all 15 trials (during acquisition the US onset occurred at a latency from the CS onset of $4 \mathrm{sec}$ ). The CR onset latency decreased asymptotically over the 15 trials, stabilizing at about $3.23 \pm 0.03 \mathrm{sec}$ (mean $\pm S E$ of the last 5 trials; Figure 3 ). The mean CR onset latency after the first trial was $3.98 \pm 0.07 \mathrm{sec}$, whereas, after the last trial, it was $3.18 \pm 0.08 \mathrm{sec}$, yield- 


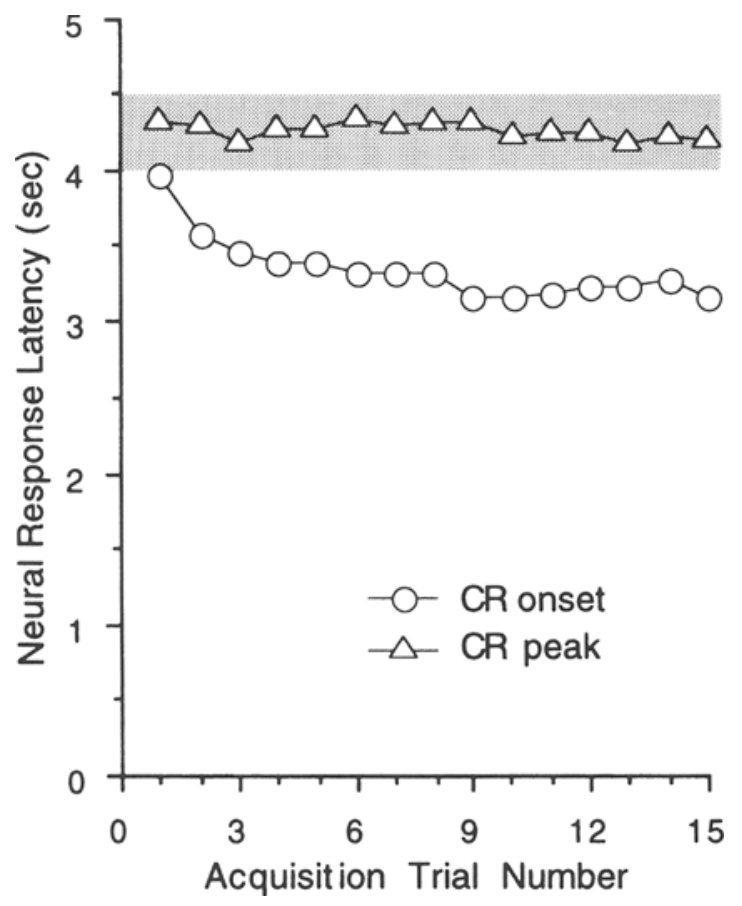

Figure 3. Latency to onset and peak of the conditioned response across acquisition trials. Using data from the same set of simulations presented in Figure 2, this plot shows the changes in the CR peak (triangles) and onset (circles) latencies (see Simulation Method section for the operational definitions) across acquisition trials. The plotted points are the values for each trial averaged across six statistically independent simulations. Error bars are not shown because they were generally too small to illustrate. Note that the latency of $C R$ peak remained constant across the 15 conditioning trials, and its latency corresponded accurately to the expected presence of the US, which is indicated by the shaded area. The CR onset normally anticipated the expected US onset (all onset data points are below the shaded area), and it decreased asymptotically across trials.

ing a mean latency decrease of $0.80 \mathrm{sec}$. An analysis of variance showed that the trial effect was statistically significant $[F(14,75)=7.03, p<.05]$. A post hoc comparison (Fisher's PLSD) revealed a significant decrease in the CR onset latency on every trial after the first one $(p<$ .05 ). This pattern of results was not unique to conditioning with a 4-sec ISI. The same pattern was also seen, in the experiments described below, using 5-, 12-, and 15-sec ISIs. In all three cases, the neural CR latency anticipated the expected time of the US onset, and the CR onset latency decreased across trials.

CR peak coincides with the expected time of the US. During the initial simulations of acquisition (described above) using a 4-sec ISI, the latency of the CR peak corresponded to the time between the US onset and offset during training (Figure 2; Figure 3 triangles). The mean $( \pm S E)$ CR peak latency across trials was $4.27 \pm$ $0.01 \mathrm{sec}$. Recall that the US onset occurred at $4.0 \mathrm{sec}$, and its offset was at $4.5 \mathrm{sec}$ (shaded area in Figure 2), giving a midpoint of $4.25 \mathrm{sec}$. In contrast to the CR onset la- tency, the latency of the CR peak did not appear to exhibit systematic changes in across trials (Figure 2; Figure 3 triangles). Not surprisingly, given the graphical data (Figure 2; Figure 3 triangles), there was no significant main effect of trials on CR peak latency $[F(14,75)=1.31, p>$ $.05]$. In the experiments described below, the same pattern of results was also seen using a 5-, 12-, or 15-sec ISI. Specifically, the latency of the peak CR occurred between the expected times of the US onset and offset, and the latency of the peak did not decline with increasing numbers of conditioning trials.

Mixed ISI training causes a double-peaked CR. After training on 5- and 15-sec ISIs on alternate trials, the model generated a double-peaked CR. The latency of each CR peak corresponded to one of the ISIs employed during training (early peak latency $=5.22 \pm 0.02 \mathrm{sec}$; late peak latency $=15.18 \pm 0.04 \mathrm{sec}$ ). The two output distributions were clearly separated by an intermediate interval within which there was no neural output. The plot in Figure 4 shows the latencies of all CS-generated output spikes in ALa. It is worthwhile calling attention to an obvious feature of the response topography revealed in Figure 4: The temporal spread of the CR associated with the 15-sec ISI is clearly larger than the temporal spread of the CR associated with the 5-sec ISI. The standard deviations of the CRs were $0.48 \pm 0.02 \mathrm{sec}$ and $0.98 \pm$ $0.06 \mathrm{sec}$, respectively, for the responses corresponding to the 5- and 15-sec ISIs. These differences in the standard deviations were statistically significant [paired $t$ test, $t(5)=7.06, p<.05]$. This finding does not seem to be an isolated case. As we shall see below, the response topography is generally ISI dependent.

Shift in training ISI causes a jump in CR latency. After 15 training trials on the 4-sec ISI, the model exhibited the expected $C R$, with a mean peak latency of $4.34 \pm$ $0.02 \mathrm{sec}$ (Figure 5Al). When the ISI was changed to $12 \mathrm{sec}$ and 15 additional conditioning trials were given, a new CR emerged with a mean peak latency of $12.15 \pm$ $0.07 \mathrm{sec}$, whereas the original peak extinguished (Figure 5A3). Examination of the intermediate trials, after the ISI shift but before the completion of conditioning, revealed that the CR peak changed as a discrete jump from the original latency to the new one, without traversing the intervening latencies. Figure 5A2 shows the $\mathrm{CR}$ after two trials of training on the second ISI. The ISI change caused partial extinction of the early CR and rapid acquisition of the later CR. There was no output at intervening latencies. Note that the response topography seems to be ISI dependent. Specifically, the temporal spread of the output is less for the original (shorter latency) CR (Figure 5A 1) than for the new (longer latency) CR (Figure 5A3). The standard deviations for the original and new CRs were $0.47 \pm 0.02 \mathrm{sec}$ and $0.75 \pm 0.06 \mathrm{sec}$, respectively-a difference that was statistically significant [paired $t$ test, $t(5)=3.90, p<.05]$.

To evaluate possible effects of the order of conditioning (direction of shift), in a separate set of simulations, the sequence of ISIs was reversed. The model was first 


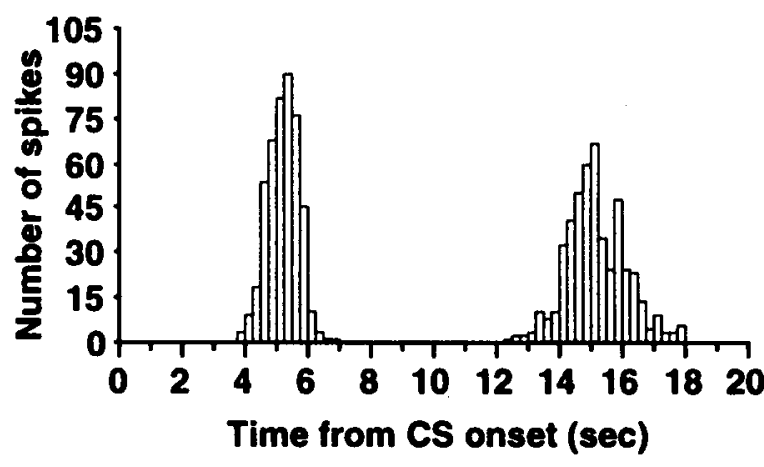

Figure 4. Temporal form of the conditioned response.(CR) following mixed ISI training. The conditioning ISI alternated between 5 and 15 sec across trials, for a total of 20 trials. The histogram shows the timing (latency from the CS onset) of action potentials (pooled across six statistically independent simulations) in the output neurons of the lateral amygdala (ALa). The neural CR was clearly double-peaked, and the timing of each peak corresponded to the expected time of the USs used in the two conditioning trial types. Note that the neural CRs associated with the two trial types are completely separated in time, with no intervening activity. Also note that the temporal spread of the CR corresponding to the longer ISI is greater than that of the shorter latency response. It is a general property of the model that longer ISIs during training result in broader averaged $C R$ waveforms.

given 15 training trials on the 12 -sec ISI, after which it exhibited a CR with a mean peak latency of $12.29 \pm$ $0.05 \mathrm{sec}$ (Figure $5 \mathrm{Bl}$ ). When the ISI was then changed to $4 \mathrm{sec}$ and 15 additional conditioning trials were given, a new CR emerged, with a mean peak latency of $4.32 \pm$ $0.01 \mathrm{sec}$, whereas the original peak extinguished (Figure 5B3). Using this reversed sequence of conditioning, the results were therefore essentially the same, except that the changes occurred in the opposite order. Examination of the intermediate trials, after the ISI shift but before completion of conditioning, again revealed that the CR peak changed as a discrete jump of the peak from the original latency to the new one, without traversing the intervening latencies. The response topography again seemed to be ISI dependent. In particular, the temporal spread was greater for the original CR (Figure 5B1) than for the new one (Figure 5B3). The standard deviations for the original and new CRs were $0.75 \pm 0.04 \mathrm{sec}$ and $0.49 \pm$ $0.01 \mathrm{sec}$, respectively - a difference that was statistically significant [paired $t$ test: $t(5)=6.48, p<.05$ ]. Comparison of the two ISI shifts (Figure 5A vs. Figure 5B) suggests that the key results do not reflect an order effect.

Temporal spread of the averaged CR waveform is ISI dependent. The results presented thus far suggest that the temporal spread of the neural CR is ISI dependent. This was first noticed in the mixed ISI conditioning (Figure 4) and was also suggested in the results obtained when the ISI was shifted (Figure 5). In the mixed ISI conditioning, the earlier of the two peaks clearly showed less temporal spread than the later one. Interestingly, the overall size of the two responses was similar across runs in terms of total number of output spikes (average early peak size $=77.0 \pm 7.9$ spikes; average late peak size $=$ $80.5 \pm 10.9$ spikes). The same general pattern was seen in the shifted-ISI simulation, regardless of the order in which the two conditioning ISIs were presented. Altogether, in the present simulations, we looked at four different ISIs $(4,5,12$, and $15 \mathrm{sec})$ that were used in a total of seven separate sets of simulations (three sets included the 4-sec ISI, two sets included the 12-sec ISI, and one set included the 5- and 15-sec ISIs), in which each set includes six replications. We analyzed these seven data points to explore further the ISI dependence of the CR topography. There was a significant $(p<.05)$ positive correlation $(r=.99)$ and an increasing monotonic relationship between the conditioning ISI and the standard deviation of the output spike latencies. These findings demonstrate that the CR topography depends on the conditioning ISI - in particular, that the temporal spread of the averaged $\mathrm{CR}$ waveform is positively correlated with the conditioning ISI.

\section{Discussion}

Our fear conditioning model makes five time-domain predictions, summarized below, regarding CR production. The model illuminates how these temporal aspects of conditioning can emerge naturally from neurobiological mechanisms, and it furnishes a computational platform for Pavlov's original notion of a slowly spreading "wave of excitation."

Temporal aspects of $C R$ production. Earlier, we discussed five facts concerning CR timing that have been documented using short-delay (short-ISI) classical conditioning procedures, noting that relatively less is known about CR timing using the longer ISIs that are common in fear conditioning studies. Here, we used computer simulations to the explore predictions of our circuit-level model of fear conditioning with regard to these five temporal aspects of CR production. These simulations used ISIs in the range of $4-15 \mathrm{sec}$. The results were in line with findings from studies using short-delay conditioning. What follows immediately below summarizes our results and cites corresponding behavioral findings based on short-delay conditioning.

First, after conditioning, the CR onset preceded the expected US onset, and the CR onset latency decreased with progressive training (see Figure 2, and Figure 3 circles; cf. Frey \& Ross, 1968; Schneiderman, 1966; Schneiderman \& Gormezano, 1964; Suboski, 1967). Second, the peak of the CR occurred during the expected time of the US, between its expected onset and offset (see Figure 2, and Figure 3 triangles; cf. Smith, 1968). Third, a doublepeaked CR was generated when training included a mixture of two different ISIs (see Figure 4; cf. Hoehler \& Leonard, 1976; Kehoe et al., 1989; Millenson et al., 1977). Fourth, when the ISI was changed abruptly during training, the latency of the CR peak exhibited a discrete jump from the time of the original US to the time of the new one (see Figure 5; cf. Boneau, 1958; Coleman \& Gormezano, 1971; Salafia et al., 1979), and there were transition tri- 

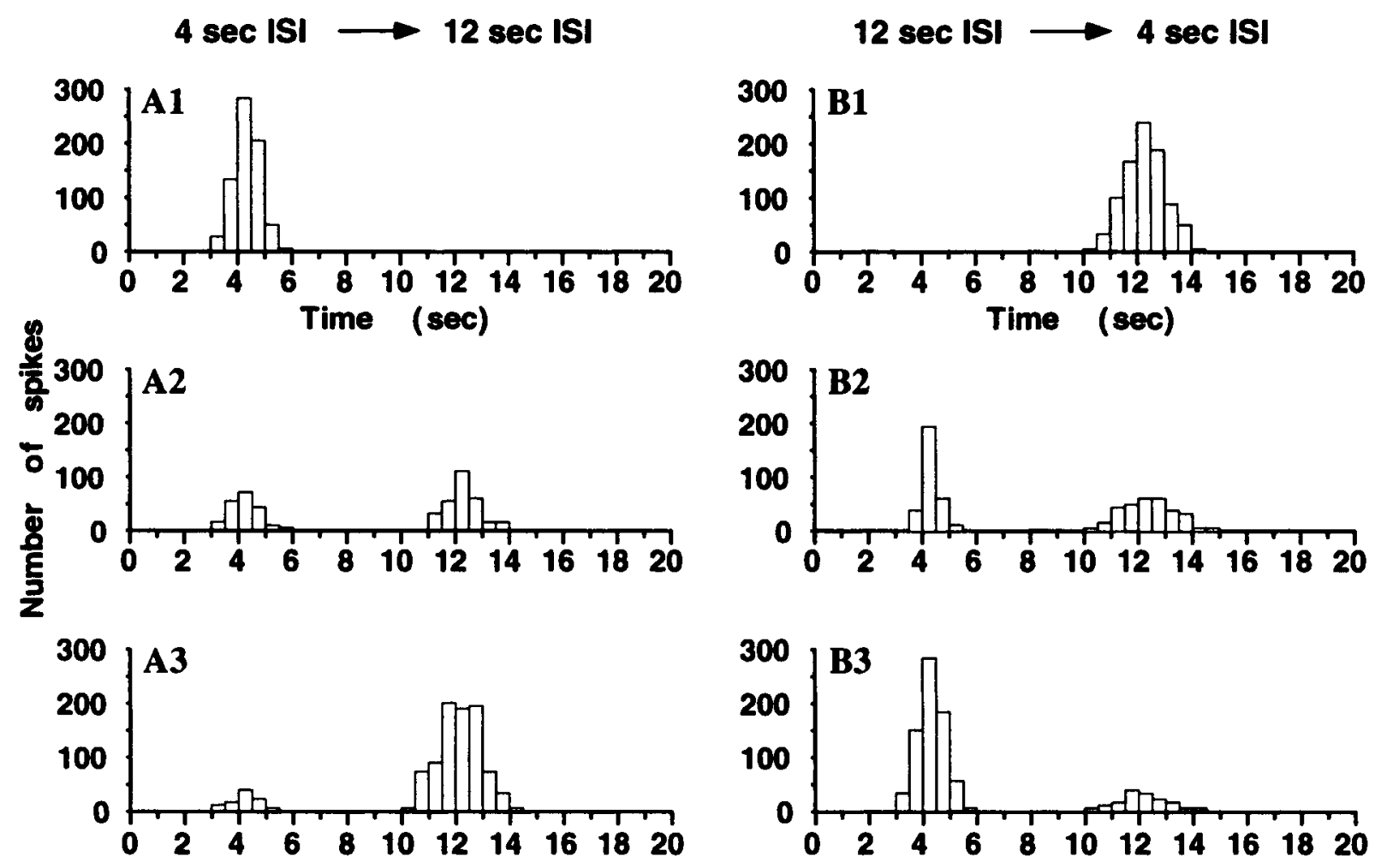

Figure 5. ISI shifts during conditioning cause discrete jumps in CR latency. Illustrated data were chosen to be representative of CR topographies following learning in each phase of the conditioning paradigm. (A) Training began with a 4-sec ISI, which was then shifted to a 12-sec ISI. A1 shows the neural CRs after 15 acquisition trials with a 4-sec ISI. A2 shows the neural CRs two trials after shifting from the 4-sec ISI to the 12-sec ISI. The early response has already begun to extinguish and a late response, timed to peak during the new US, is starting to emerge. A3 shows the response topography after 15 conditioning trials with the 12 -sec ISI. The shortlatency ISI has largely extinguished, and a longer latency $C R$ is now prominent. A1-A3 sequence helps illustrate the fact that the latency of the $C R$ peak jumps discretely from the original latency to the new latency without traversing the intervening range. (B) The conditioning ISI order was reversed. Specifically, conditioning began with a 12-sec ISI and was then shifted to a 4-sec ISI. B1 shows the CRs after 15 trials conditioning trials with a 12-sec ISI. B2 shows the neural CR two trials after shifting from the 12-sec ISI to the 4-sec ISI. The late response has begun to extinguish and a new CR peak is beginning to develop at the time of the new ISI. B3 shows the neural CR after 15 conditioning trials using the 4-sec ISI. The long-latency CR has largely extinguished, and an early response, timed to peak during the new US, is now prominent. B1-B3 sequence helps illustrate the fact that the latency of the CR peak jumps discretely from the original latency to the new latency. The pattern of the results appears to be the same regardless of the order of conditioning.

als in which double-peaked CRs (corresponding to the two ISIs) were evident (see Figure 5; cf. Coleman \& Gormezano, 1971; Leonard \& Theios, 1967). Fifth, the temporal spread of the averaged CR waveform was positively correlated with the conditioning ISI (see Figures 4 and 5; cf. Smith, 1968).

In summary, the model predicts that the same temporal properties of $\mathrm{CR}$ production that have been observed in short-delay conditioning should also be observed in long-delay conditioning, including fear conditioning, provided one uses a response measure with appropriate temporal resolution. The paradigm developed by J. S. Brown et al. (1951), involving conditioned enhancement of an unconditioned response, could prove useful in this regard (see Davis et al., 1989; Lam et al., 1996; Siegel, 1967; Weisz \& Walts, 1990).

Circuit-level mechanism of anticipatory CRs. Our model is useful for elucidating a possible neurobiological mechanism by which the CR onset is caused to precede the US onset (Figure 2; Figure 3 circles). The mechanism involves the potentiation of synapses whose time-locked activity windows largely precede but partially overlap the US. When these synapses are sufficiently potentiated to fire the ALa output neurons, they do so for the entire duration of their activity window, including the part that preceded the US. Firing of these output neurons produces a CR in advance of the US onset. Synapses whose windows only partially overlap the US (or that only overlap the US on some trials because of temporal variability produced by the synaptic noise) are potentiated less each trial than those whose windows overlap the US more completely. Over trials, this slower rate of synaptic potentiation causes a gradual recruitment of early-firing output neurons in ALa into the pool that generates the CR. Gradual recruitment of these early-firing ALa neurons in turn causes a progressive decrease in CR onset latency. In the 
current simulations, the temporal limits of this anticipatory response are determined by the length $(\sim 0.8 \mathrm{sec})$ and number of the activity windows (see Tieu et al., 1999), both of which are limited in practice by our current computational overhead.

Multiple independent loci for learning each ISI. Our model suggests a possible common mechanism for the results observed using either a mixed ISI (Figure 4) or a shifted ISI (Figure 5) conditioning paradigm. Recall that alternating the ISI on sequential conditioning trials caused a double-peaked CR (see Figure 4), and shifting the ISI during conditioning caused the CR peak to jump to the time of the new US (see Figure 5). It is easy to see how and why this occurs in the model. A fundamental characteristic of the circuit architecture is that each plastic synapse codes for a specific range of delay from the CS onset (its "activity window"; see Tieu et al., 1999). Each activity window overlaps several others with similar latencies, thus allowing multiple synapses to encode a single ISI. Note that the activity windows are brief $(\sim 0.8 \mathrm{sec})$ relative to the range of delays the model has been shown to encode $(0.5-16.0 \mathrm{sec})$. This means that learning about multiple ISIs (whose difference is greater than the duration of the activity windows) will occur at different synapses (at different physical locations).

The generation of double-peaked CRs (see Figure 4) and discrete jumps in CR latency (see Figure 5) thus results from the independent, parallel potentiation and depression of ISI-specific sets of synapses. This mechanism is reminiscent of an interpretation given to a human eyeblink conditioning study that involved a shifted ISI paradigm (Boneau, 1958). This eyeblink study compared the development of CRs in the postshift phase of the conditioning procedure to $\mathrm{CR}$ development in naive subjects conditioned at the postshift ISI. The conclusion was that "the responses appropriate to the two interstimulus intervals thus seem to demonstrate independence, one extinguishing while the other shows acquisition, with no evidence of interaction between the two processes" (Boneau, $1958, p .469)$. This independence is a clear prediction of our model, provided that the difference between the ISIs is substantial. With combinations of similar ISIs in which some single activity windows overlap both times, the responses cease to be independent, resulting in a blurring together of the CRs and serial-order effects in conditioning. Our model therefore suggests that the behavioral results seen with these two different conditioning paradigms may reflect a common underlying neurobiological mechanism.

Mechanism for ISI dependence of CR topography. The model offers a plausible explanation for the fact that the CR topography depends on the conditioning ISI. Specifically, the simulations showed that the temporal spread of the neural CR is positively correlated with the ISI used during conditioning (see Figures 4 and 5), in accordance with behavioral results (Smith, 1968, Figure 5; see also Gormezano, 1972, Figure 10). The ISI depen- dence of the CR topography was expected on the basis of our previous simulations (Tieu et al., 1999). These previous results showed that the circuit encodes and learns time in a Weber-like fashion. A modern interpretation of Weber's law (Weber, 1851) in the time domain (Gibbon, 1977; Gibbon et al., 1997) is that the variability of the response latency $(\sigma)$ is proportional to the mean response latency $(\mu)$, such that the coefficient of variation is relatively constant ( $c \nu=\sigma / \mu=$ constant). Indeed, there is a substantial body of data in support of this generalization (Gibbon, 1977). One hypothesis is that the Weber law (for time) results from underlying random variation in a noise variable (Gibbon et al., 1997). Our previous simulations (Tieu et al., 1999) supported this hypothesis. In particular, we found that adding synaptic noise to the circuit did in fact cause temporal learning to follow a Weber-like law.

The ISI dependence of the CR topography seen in the present study can therefore be explained by two facts. The first is that the CR peak latency is ISI dependent, meaning that the neural CR accurately predicts the expected time of the US (Figures 2-5; see also Figure 9 of Tieu et al., 1999). The second is that synaptic noise causes the temporal spread of the neural CR to be proportional to the CR peak latency (see Figure 9 of Tieu et al., 1999), meaning that temporal learning conforms to a Weberlike law. Taken together, these two facts predict that the response variability should be proportional to the conditioning ISI, which is what was observed. It is perhaps worth noting that the accumulation of noise-produced latency variance in the model affects both learning and performance.

Computational platform for Pavlov's wave of excitation. In treating the mechanism of temporal learning, some theories rely on the theoretical construct of a stimulus trace, a time-varying internal representation of an external stimulus (Hull, 1943, 1952; Pavlov, 1927; Wagner \& Brandon, 1989). Pavlov speculated that the stimulus trace elicited by the CS was a slowly spreading wave of excitation through cortex (Pavlov, 1932, p. 93) that put neurons into different states, thus creating an intrinsically time-varying internal representation. Pavlov's idea of a slow wave of excitation was sharply criticized at the time by Guthrie (1930) on the grounds that there was no apparent way to instantiate the long internal latencies required by Pavlov's explanation. We have shown here that Pavlov's essential notion can indeed be captured in a neurobiologically inspired computational form and that this network architecture is sufficient to predict many of the temporal phenomena that Pavlov and others observed, including temporal learning at long delays and the temporal form of the CR.

\section{REFERENCES}

Applegate, C. D.. Kapp, B. S., Underwood, M. D., \& MCNall. C. L. (1983). Autonomic and somatomotor effects of amygdala central nucleus stimulation in awake rabbits. Physiology \& Behavior, 31, 353360 . 
Barrionuevo, G., \& Brown, T. H. (1983). Associative long-term synaptic potentiation in hippocampal slices. Proceedings of the $\mathrm{Na}$ tional Academy of Sciences, 80, 7347-7351.

Beggs, J. M., Brown, T. H., Byrne, J. H., Crow, T., LeDoux, J. E., LeBar, K., \& Thompson, R. F. (1999). Fundamental neuroscience. In M. J. Zigmond, F. E. Bloom, S. C. Landis, J. L. Roberts, \& L. R. Squire (Eds.), Fundamental neuroscience (pp. 1411-1454). San Diego: Academic Press.

Beggs, J. M., Moyer, J. R., McGann, J. P., \& Brown, T. H. (2000). Prolonged synaptic integration in perirhinal cortical neurons. Journal of Neurophysiology, 83, 3294-3298.

Bevins, R. A., \& AYREs, J. J. B. (1995). One-trial context fear conditioning as a function of the interstimulus interval. Animal Learning \& Behavior, 23, 400-410.

Bienenstock, E. L., CoOper, L. N., \& Monro, P. W. (1982). Theory for the development of neuron selectivity: Orientation specificity and binocular interaction in visual cortex. Journal of Neuroscience, 2, 32-48.

BONEAU, C. A. (1958). The interstimulus interval and the latency of conditioned eyelid response. Journal of Experimental Psychology, 56, 464-472.

Brown, J. S., Kalish, H. I., \& Farber, I. E. (1951). Conditioned fear as revealed by magnitude of startle response to an auditory stimulus. Journal of Experimental Psychology, 41, 317-328.

Brown, T. H., \& FAULKNER, B. (1997). Hebbian synapses. In G. Adelman \& B. Smith (Eds.), Encyclopedia of neuroscience (pp. 865-868). Amsterdam: Elsevier.

Brown, T. H., Kairiss, E. W., \& Keenan, C. L. (1990). Hebbian synapses: Biophysical mechanisms and algorithms. Annual Review of Neuroscience, 13, 475-511.

CANLI, T., \& Brown, T. H. (1996). Amygdala stimulation enhances the rat eyeblink reflex through a short-latency mechanism. Behavioral Neuroscience, 110, 51-59.

Chapman, P. F., Kairiss, E. W., Keenan, C. L., \& Brown, T. H. (1990). Long-term synaptic potentiation in the amygdala. Synapse, 6, 271278 .

ChurCh, R. M., \& Black, A. H. (1958). Latency of the conditioned heart rate as a function of the CS-US interval. Journal of Comparative \& Physiological Psychology, 51, 478-482.

Colavita, F. B. (1965). Dual function of the US in classical salivary conditioning. Journal of Comparative \& Physiological Psychology, 60, 218-222.

Coleman, S. R., \& Gormezano, I. (1971). Classical conditioning of the rabbit's (Oryctolagus cuniculus) nictitating membrane response under symmetrical CS-US interval shifts. Journal of Comparative \& Physiological Psychology, 77, 447-455.

Davis, M., Schlesinger, L. S., \& Sorenson, C. A. (1989). Temporal specificity of fear conditioning: Effects of different conditioned stimulus-unconditioned stimulus intervals on the fear-potentiated startle effect. Journal of Experimental Psychology: Animal Behavior Processes, 15, 295-310.

Ellison, G. D. (1964). Differential salivary conditioning to traces. Journal of Comparative \& Physiological Psychology, 57, 373-380.

ESTES, W. K., \& SKINNER, B. F. (1941). Some quantitative properties of anxiety. Journal of Experimental Psychology, 29, 390-400.

FANSELOW, M. S., \& LeDouX, J. E. (1999). Why we think plasticity underlying Pavlovian fear conditioning occurs in the basolateral amygdala. Neuron, 23, 229-232.

FAULKNER, B., \& Brown, T. H. (1999). Morphology and physiology of neurons in the rat perirhinal-lateral amygdala area. Journal of Comparative Neurology, 411, 613-642.

Faulkner, B., TieU, K. H., \& Brown, T. H. (1997). Mechanism for temporal encoding in fear conditioning: Delay lines in perirhinal cortex and lateral amygdala. In J. Bower (Ed.), Computational neuroscience: Trends in research 1997 (pp. 641-645). New York: Plenum.

FrEY, P. W., \& Ross, L. E. (1968). Classical conditioning of the rabbit eyelid response as a function of the interstimulus interval. Journal of Comparative \& Physiological Psychology, 65, 246-250.

GibBon, J. (1977). Scalar expectancy theory and Weber's law in animal timing. Psychological Review, 84, 279-325.

Gibbon, J., Malapani, C., Dale, C. L., \& Gallistel, C. R. (1997).
Toward a neurobiology of temporal cognition: Advances and challenges. Current Opinion in Neurobiology, 7, 170-184.

GoRmEZANO, I. (1972). Investigations of defense and reward conditioning in the rabbit. In A. H. Black \& W. F. Prokasy (Eds.), Classical conditioning II: Current research and theory (pp. 151-181). New York: Appleton-Century-Crofts

Guthrie, E. R. (1930). Conditioning as a principle of learning. Psychological Review, 37, 412-428.

HoEHLER, F. K., \& LEONARD, D. W. (1976). Double responding in classical nictitating membrane conditioning with single-CS dual-ISI training. Pavlovian Journal of Biological Sciences, 11, 180-190.

Hull, C. L. (1943). Principles of behavior. New York: Appleton-CenturyCrofts.

HuLl, C. L. (1952). A behavior system. New Haven, CT: Yale University Press.

Kapp, B. S., Frysinger, R. C., Gallagher, M., \& Haselton, J. R. (1979). Amygdala central nucleus lesions: Effect on heart rate conditioning in the rabbit. Physiology \& Behavior, 23, 1109-1117.

KapP, B. S., Whalen, P. J., Supple, W. F., \& Pascoe, J. P. (1992). Amygdaloid contributions to conditioned arousal and sensory information processing. In J. Aggleton (Ed.), The amygdala: Neurobiological aspects of emotion, memory, and mental dysfunction (pp. 229254). New York: Wiley-Liss.

Kehoe, E. J., Graham-Clarke, P., \& Schreurs, B. G. (1989). Temporal patterns of the rabbit's nictitating membrane response to compound and component stimuli under mixed CS-US intervals. Behavioral Neuroscience, 103, 283-295.

Kehoe, E. J., \& Macrae, M. (1998). Classical conditioning. In W. O'Donohue (Ed.), Learning and behavior therapy (pp. 36-58). Boston: Allyn \& Bacon.

Kelso, S. R., \& Brown, T. H. (1986). Differential conditioning of associative synaptic enhancement in hippocampal brain slices. Science, 232, 85-87.

Kelso, S. R., Ganong, A. H., \& Brown, T. H. (1986). Hebbian synapses in hippocampus. Proceedings of the National Academy of Sciences, 83, 5326-5330.

Kimble, G. A., \& ReYnolds, B. (1967). Eyelid conditioning as a function of the interval between conditioned and unconditioned stimuli. In G. Kimble (Ed.), Foundations of conditioning and learning (pp. 279 287). New York: Appleton-Century-Crofts.

Krettek, J. E., \& Price, J. L. (1978). A description of the amygdaloid complex in the rat and cat with observations on intra-amygdaloid ax onal connections. Journal of Comparative Neurology, 178, 255-280.

LAM, Y.-W., Wong, A., CANLI, T., \& Brown, T. H. (1996). Conditioned enhancement of the early component of the rat eyeblink reflex. $\mathrm{Neu}$ robiology of Learning \& Memory, 66, 212-220.

LeDoux, J. E. (1995). Emotion: Clues from the brain. Annual Review of Psychology, 46, 209-235.

LEONARD, D. W., \& THEios, J. (1967). Effect of CS-US interval shift on classical conditioning of the nictitating membrane in the rabbit. Journal of Comparative \& Physiological Psychology, 63, 355-358.

LeVY, W. B., \& STEWART, O. (1979). Synapses as associative memory elements in the hippocampal formation. Brain Research, 175, 233-245.

LibBY, M. E., \& ChURCH, R. M. (1975). Fear gradients as a function of the temporal interval between signal and aversive event in the rat. Journal of Comparative \& Physiological Psychology, 88, 911-916.

Maes, J. H. R., \& Vossen, J. M. H. (1992). One-trial aversive conditioning to contextual cues: Effects of time of shock presentation on freezing during conditioning and testing. Bulletin of the Psychonomic Society, 30, 403-406.

MAGEE, J. C., \& Johnston, D. (1997). A synaptically controlled, associative signal for Hebbian plasticity in hippocampal neurons. Science, 275, 209-213.

MALENKA, R. C. (1995). LTP and LTD: Dynamic and interactive processes of synaptic plasticity. The Neuroscientist, 1, 35-42.

MAREN, S. (1996). Synaptic transmission and plasticity in the amygdala. Molecular Neurobiology, 13, 1-22.

MAREN, S. (1999). Long-term potentiation in the amygdala: A mechanism for emotional learning and memory. Trends in Neurosciences, 22, 561-567. 
Maren, S., \& Fanselow, M. S. (1996). The amygdala and fear conditioning: Has the nut been cracked? Neuron, 16, 237-240.

Mauk, M. D., \& RuIZ, B. P. (1992). Learning-dependent timing of Pavlovian eyelid responses: Differential conditioning using multiple interstimulus intervals. Behavioral Neuroscience, 106, 666-681.

Millenson, J. R., Kehoe, E. J., \& Gormezano, I. (1977). Classical conditioning of the rabbit's nictitating membrane response under fixed and mixed CS-US intervals. Learning \& Motivation, 8, 351 366.

Moore, J. W., \& ChOI, J.-S. (1997). Conditioned response timing and integration in the cerebellum. Learning \& Memory, 4, 116-129.

Pavlov, I. P. (1927). Conditioned reflexes (G. V. Anrep, Trans.). London: Oxford University Press.

Pavlov, I. P. (1932). The reply of a physiologist to psychologists. Psychological Review, 39, 91-127.

QUiRK, G. J., RePA, C., \& LeDoux, J. E. (1995). Fear conditioning enhances short-latency auditory responses of lateral amygdala neurons: Parallel recordings in the freely behaving rat. Neuron, 15, 1029-1039.

Romanski, L. M., Clugnet, M.-C., Bordi, F., \& LeDoux, J. E. (1993). Somatosensory and auditory convergence in the lateral nucleus of the amygdala. Behavioral Neuroscience, 107, 444-450.

Salafia, W. R., Martino, L. J., Cloutman, K., \& Romano, A. G. (1979). Unconditional-stimulus locus and interstimulus-interval shift in rabbit (Oryctolagus cuniculus) nictitating membrane conditioning. Pavlovian Journal of Biological Science, 14, 64-71.

SCHNEIDERman, N. (1966). Interstimulus interval function of the nictitating membrane response of the rabbit under delay versus trace conditioning. Journal of Comparative \& Physiological Psychology. 62 , 397-402.

Schneiderman, N. (1972). Response system divergencies in aversive classical conditioning. In A. H. Black \& W. F. Prokasy (Eds.), Classical conditioning II: Current research and theory (pp. 341-376). New York: Appleton-Century-Crofts.

Schneiderman. N.. \& Gormezano, 1. (1964). Conditioning of the nictitating membrane of the rabbit as a function of CS-US interval. Journal of Comparative \& Physiological Psychology, 57, 188-195.

SHI. C.. \& Davis. M. (1999). Pain pathways involved in fear conditioning measured with fear-potentiated startle: Lesion studies. Journal of Neuroscience, 19, 420-430.

SiEgEL, A. (1967). Stimulus generalization of a classically conditioned response along a temporal dimension. Journal of Comparative \& Physiological Psychology, 64, 461-466.

SMITH, M. C. (1968). CS-US interval and US intensity in classical conditioning of the rabbit's nictitating membrane response. Journal of Comparative \& Physiological Psychologv. 66, 679-687.

Stefanacci, L., Farb, C. R., Pitkänen, A., Go, G., LeDoux, J. E., \& Amaral, D. G. (1992). Projections from the lateral nucleus to the basal nucleus of the amygdala: $A$ light and electron microscopic PHA-L study in the rat. Journal of Comparative Neurology, 323, 586-601.

SUBosKI, M. D. (1967). UCS intensity and the latency of the classically conditioned eyelid response. Journal of Experimental Psychology, 74, 31-35.

THOMPSON, R. F. (1988). The neural basis of basic associative learning of discrete behavioral responses. Trends in Neurosciences, 11, 152-155.

Thompson, R. F., \& KRUPA, D. J. (1994). Organization of memory traces in the mammalian brain. Annual Review of Neuroscience, 17, 519-549.

Tieu, K. H., Keidel, A. L., McGann, J. P., Faulkner, B., \& Brown, T. H. (1999). Perirhinal-amygdala circuit-level computational model of temporal encoding in fear conditioning. Psychobiology, 27, 1-25.

Wagner, A. R., \& Brandon, S. E. (1989). Evolution of a structured connectionist model of Pavlovian conditioning (AESOP). In S. B. Klein \& R. R. Mowrer (Eds.), Contemporary learning theories: Pavlovian conditioning and the status of traditional learning theory (pp. 149-189). Hillsdale, NJ: Erlbaum.

WeBER, E. H. (1851). Annotationes anatomicae et physiologicae [Anatomical and physiological annotations]. Leipzig: C. F. Koehler.

Weisz, D. J., HARDEN. D., \& XiANG, Z. (1992). Effects of amygdala lesions on reflex facilitation and conditioned response acquisition during nictitating membrane response conditioning in the rabbit. Behavioral Neuroscience, 106, 262-273.

WeISZ, D. J., \& LoTurCo, J. (1988). Reflex facilitation of the nictitating membrane response remains after cerebellar lesions. Behavioral Neuroscience, 102, 203-209.

WEISZ, D. J., \& MCINERNEY, J. (1990). An associative process maintains reflex facilitation of the unconditioned nictitating membrane response during the early stages of training. Behavioral Neuroscience, 104, $21-27$

WEISZ, D. J., \& WALTS, C. (1990). Reflex facilitation of the rabbit nictitating membrane response by an auditory stimulus as a function of interstimulus interval. Behavioral Neuroscience, 104, 11-20.

\section{NOTE}

1. The values of $n_{1}$ and $n_{2}$ were reduced to $3.0 \times 10^{-5}$ and $4.0 \times$ $10^{-7}$, respectively, and the value of $\theta_{d}$ was reduced to 0 . All three of these constants relate to the relative rates of LTP and LTD at plastic synapses. See Appendices D and E of Tieu et al. (1999) for a detailed explanation of these parameters.

(Manuscript received August 4, 1999; accepted for publication October 19, 1999.) 